Research Article

\title{
Isolation and characterization of genes functionally involved in ovarian development of the giant tiger shrimp Penaeus monodon by suppression subtractive hybridization (SSH)
}

\author{
Rachanimuk Preechaphol $^{1^{*} \text {, Sirawut Klinbunga }}{ }^{2,3}$, Bavornlak Khamnamtong ${ }^{2,3}$ and Piamsak Menasveta ${ }^{2,4}$ \\ ${ }^{1}$ Program in Biotechnology, Faculty of Science, Chulalongkorn University, Bangkok, Thailand. \\ ${ }^{2}$ Center of Excellence for Marine Biotechnology, Faculty of Science, Chulalongkorn University, \\ Bangkok, Thailand. \\ ${ }^{3}$ Aquatic Molecular Genetics and Biotechnology Laboratory, National Center for Genetic Engineering \\ and Biotechnology, National Science and Technology Development Agency, Pathumthani, Thailand. \\ ${ }^{4}$ Department of Marine Science, Faculty of Science, Chulalongkorn University, Bangkok, Thailand.
}

\begin{abstract}
Suppression subtractive hybridization (SSH) libraries between cDNA in stages I (previtellogenic) and III (cortical rod) ovaries of the giant tiger shrimp (Penaeus monodon) were established. In all, 452 ESTs were unidirectionally sequenced. Sequence assembly generated 28 contigs and 201 singletons, 109 of which (48.0\%) corresponding to known sequences previously deposited in GenBank. Several reproduction-related transcripts were identified. The full-length cDNA of anaphase promoting complex subunit 11 (PmAPC11; 600 bp with an ORF of 255 bp corresponding to a polypeptide of 84 amino acids) and selenoprotein M precursor (PmSePM; 904 bp with an ORF of 396 bp corresponding to a polypeptide of 131 amino acids) were characterized and reported for the first time in penaeid shrimp. Semiquantitative RT-PCR revealed that the expression levels of PmSePM and keratinocyte-associated protein 2 significantly diminished throughout ovarian development, whereas Ser/Thr checkpoint kinase 1 (Chk1), DNA replication licensing factor mcm2 and egalitarian were down-regulated in mature ovaries of wild $P$. monodon $(p<0.05)$. Accordingly, the expression profiles of PmSePM and keratinocyte-associated protein 2 could be used as biomarkers for evaluating the degree of reproductive maturation in domesticated $P$. monodon.
\end{abstract}

Key words: EST, SSH, Penaeus monodon, ovarian development, semiquantitative RT-PCR.

Received: December 14, 2009; Accepted: June 29, 2010.

\section{Introduction}

The giant tiger shrimp (Penaeus monodon) is one of the most economically important cultured species (BaileyBrock and Moss, 1992; Rosenberry, 2001). Breeding $P$. monodon in captivity, besides being difficult (Withyachumnarnkul et al., 1998; Wongprasert et al., 2006), is very much restricted by the current dependency on wild-caught broodstock, with the consequential overexploitation of high-quality sources in the wild. As a result, aquacultural production of $P$. monodon has undergone a significant decline over the last several years (Limsuwan, 2004).

The low degree of reproductive maturation of captive $P$. monodon has also limited the ability to genetically im-

Send correspondence to Sirawut Klinbunga. Aquatic Molecular Genetics and Biotechnology Laboratory, National Center for Genetic Engineering and Biotechnology, National Science and Technology Development Agency, 113 Paholyothin Road, Klong 1, Klong Luang, Pathumthani 12120, Thailand. E-mail: sirawut@biotec.or.th. "Present address: Faculty of Marine Technology, Burapha University, Chanthaburi Campus, Chanthaburi 22170, Thailand. prove this important species by domestication and selective breeding programs (Withyachumnarnkul et al., 1998; Kenway et al., 2006; Preechaphol et al., 2007). Eyestalk ablation is used commercially to induce ovarian maturation in penaeid shrimp but the technique leads to an eventual loss in egg quality and death of the spawners (Benzie, 1998). Therefore, predictable maturation and spawning of captive penaeid shrimp without the use of eyestalk ablation is a long-term goal for the industry (Quackenbush, 1992).

Basic information on ovarian development is somewhat limited in this shrimp. Initial steps towards an understanding of the molecular mechanisms involved in ovarian and oocyte development in this economically important species, are the identification and characterization of genes differentially expressed in the diverse stages of the process (Preechaphol et al., 2007).

Recently, genes expressed in the shrimp's vitellogenic ovaries were identified and characterized. A total of 1051 clones from a conventional cDNA library were unidirectionally sequenced from the 5 ' terminus. The nucleo- 
tide sequences of 743 EST (70.7\%) significantly matched known genes previously deposited in GenBank ( $E$-value $<$ $\left.10^{-4}\right)$, whereas 308 ESTs $(29.3 \%)$ were regarded as newly unidentified transcripts $\left(E\right.$-value $\left.>10^{-4}\right)$. A total of 559 transcripts (87 contigs and 472 singletons) were obtained after sequence assembly. Several reproduction-related genes, viz., chromobox protein, ovarian lipoprotein receptor, progestin membrane receptor component 1 and ubiquitin-specific proteinase 9, X chromosome, were isolated and characterized (Preechaphol et al., 2007).

Suppression subtractive hybridization ( $\mathrm{SSH}$ ) is widely used for isolating differentially expressed genes in any two closely related samples, specimens or species (Diatchenko et al., 1996). This technique should facilitate the identification of genes involved in ovarian (and oocyte) development. The genes identified could further assist in the domestication and selective breeding programs of $P$. monodon.

In order to provide a further insight into the molecular mechanisms involved in the reproductive maturation processes of $P$. monodon, we carried out SSH of genes expressed in stages I and III ovaries of wild $P$. monodon. The expression profiles of five reproduction-related genes during ovarian development in wild $P$. monodon broodstock were further examined using semiquantitative RT-PCR. Candidate biomarkers for evaluating the degrees of reproductive maturation in captive shrimp are reported herein.

\section{Materials and Methods}

\section{Experimental animals}

Four-month-old juveniles of $P$. monodon, with body weights of approximately $25-30 \mathrm{~g}$, were purchased from a commercial farm in Chachoengsao (eastern Thailand). These were cultured in $15 \mathrm{ppt}$ seawater at ambient temperature $\left(28-32{ }^{\circ} \mathrm{C}\right)$ and a natural daylight cycle. Broodstock shrimp, with body weights of $>200 \mathrm{~g}$, were wild-caught from Satun, located in the Andaman Sea, west of peninsular Thailand. Prior to SSH library construction, ovaries were dissected out from two broodstock and weighed. The gonadosomatic index (GSI), i.e., ovarian weight/body weight $\mathrm{x} 100$, of each shrimp was calculated. In order to determine expression profiles of reproduction-related genes during $P$. monodon ovarian development, female juveniles and the broodstock were acclimated at normal farm conditions $\left(28-30{ }^{\circ} \mathrm{C}\right.$, natural daylight and $35 \mathrm{ppt}$ seawater) for 2-3 days. Ovarian developmental stages of broodstock were classified according to GSI: $<1.5,2-4$, $>4-6$ and $>6 \%$ for previtellogenic (I), vitellogenic (II), early cortical rod (III) and mature (IV) ovaries ( $N=4$ for each stage), respectively. Ovaries were dissected from each shrimp immediately after collection and kept at $-80{ }^{\circ} \mathrm{C}$ until use.

\section{Isolation of total RNA and mRNA}

Total RNA was extracted from various tissues of each individual with TRI-Reagent (Molecular Research Center) and mRNA was further purified using a QuickPrep Micro mRNA Purification Kit (GE Healthcare). Total RNA and mRNA were kept under absolute ethanol at $-80{ }^{\circ} \mathrm{C}$, prior to reverse transcription.

\section{Construction of suppression subtractive} hybridization (SSH) cDNA libraries and EST analysis

Initially, two micrograms of mRNA from the ovaries of the P. monodon broodstock were reverse-transcribed. Suppression subtractive hybridization ( $\mathrm{SSH}$ ) between cDNA from stages III $(\mathrm{GSI}=5.69 \%)$ and I $(1.43 \%)$ and vice versa (Diatchenko et al., 1996) was carried out using a PCR-Select cDNA Subtraction Kit (BD Clontech). The subsequent products were ligated to pGEM-T Easy vector and transformed into E. coli JM109. Plasmid DNA was extracted from clones carrying $>300 \mathrm{bp}$ inserts and unidirectionally sequenced using the M13 reverse primer. Sequencing data were pre-processed to remove low-quality sequences (sequence length $<100 \mathrm{bp}$, the percentage of undetermined bases $>3 \%$ and low complexity), by using SeqClean with option-A to disable the trimming of poly A tail. Repetitive sequences matching the RepBase dataset were masked by using RepeatMasker. Sequence clustering and assembly was done using TIGR Gene-Indices Clustering Tools (TGICL) (Pertea et al., 2003) with CAP3 (Huang and Madan, 1999). Nucleotide sequences of assembled and non-assembled ESTs were compared with GenBank data using BlastN and BlastX (Altschul et al., 1990). Significantly matches to nucleotides/proteins were considered when the $E$-value was $<1 \times 10^{-4}$. Blast2GO was used for the additional annotation of biological activities in BlastX matched sequences, thereby enabling Gene Ontology (GO) prediction of sequence data for which no GO annotation is, as yet, available (Conesa et al., 2005).

ESTs representing $P$. monodon selenoprotein $M$ precursor (PmSePM) and anaphase promoting complex subunit 11 (PmAPC11) were further sequenced from the reverse direction of the original cDNA clones by using a M13 forward primer.

\section{Semiquantitative RT-PCR}

Expression profiles of keratinocyte-associated protein 2, Ser/Thr checkpoint kinase 1, DNA replication licensing factor mcm2, PmSePM and egalitarian during ovarian development of $P$. monodon broodstock were analyzed by way of semiquantitative RT-PCR. EF-1 $\alpha$ was included as the positive control. Initially, nonquantitative RT-PCR (Klinbunga et al., 2009) was carried out using $100 \mathrm{ng}$ of first-strand cDNA as the template, with varying concentrations of primers $(0.05,0.10,0.15,0.20,0.25,0.30$ and $0.40 \mu \mathrm{M}$, respectively). Primer sequences are listed in 
Table 1 - Nucleotide sequences of primers used for expression analysis of keratinocyte-associated protein 2, Ser/Thr checkpoint kinase 1, DNA replication licensing factor mcm2, selenoprotein M precursor and egalitarian in ovaries of wild P. monodon broodstock.

\begin{tabular}{|c|c|}
\hline Gene & Primer sequence \\
\hline \multirow[t]{2}{*}{ Keratinocyte-associated protein 2} & F: 5'-CTGCTGTAAACAATCTGGAAAAC-3' \\
\hline & R: 5'-GGGACACCTGAGCGGAAGT-3' \\
\hline \multirow[t]{2}{*}{ Ser/Thr checkpoint kinase 1 (Chkl) } & F: 5'-CTCCCCAGTGTCCTTATTGATTAG-3' \\
\hline & R: 5'-TGGCTTTCATTCCCTCGCTG-3' \\
\hline \multirow[t]{2}{*}{$D N A$ replication licensing factor $m \mathrm{~cm} 2$} & F: 5'-TCAAGCGAGACAACAACGAACT-3' \\
\hline & R: 5'-TTGGACCATCACTGGGCATC-3' \\
\hline \multirow[t]{2}{*}{ Selenoprotein M precursor (PmSePM) } & F: 5'-GACATCCCACTCTTCCATAAT-3' \\
\hline & R: 5'-TTTCATCTACAGTTCTTCCCTC-3' \\
\hline \multirow[t]{2}{*}{ Egalitarian } & F: 5'-CACTTGTGCCCACTGTCTATG-3' \\
\hline & R: 5'-CCTCCACTGCCAACACTACTC-3' \\
\hline \multirow[t]{2}{*}{$E F-1 \alpha$} & F: 5'-ATGGTTGTCAACTTTGCCCC-3' \\
\hline & R: 5'-TTGACCTCCTTGATCACACC-3' \\
\hline
\end{tabular}

Table 1. Optimal concentrations of $\mathrm{MgCl}_{2}(1.0,1.5,2.0$, and $3.0 \mathrm{mM}$ ) were further selected using an optimized primer concentration. Finally, RT-PCR of these genes was undertaken with an optimized primer and $\mathrm{MgCl}_{2}$ concentrations for 20, 22, 24, 27, 30 and 35 cycles. The number of cycles before the product reached an amplification plateau was chosen.

Semiquantitative RT-PCR was undertaken with $1.5 \mathrm{mM}$ of $\mathrm{MgCl}_{2}$ and $0.2 \mu \mathrm{M}$ of primers for the respective target genes, $0.15 \mu \mathrm{M}$ of primers for egalitarian and $0.10 \mu \mathrm{M}$ of those for $E F$ 1- $\alpha$, as follows: $94{ }^{\circ} \mathrm{C}$ for $3 \mathrm{~min}$ followed by appropriate cycles $(22,27,24,22$ and 24 cycles for the target genes and 22 cycles for $E F$ 1- $\alpha$, respectively) of $94{ }^{\circ} \mathrm{C}$ for $30 \mathrm{~s}, 53{ }^{\circ} \mathrm{C}$ for $45 \mathrm{~s}$ and $72{ }^{\circ} \mathrm{C}$ for $45 \mathrm{~s}$ and a final extension at $72{ }^{\circ} \mathrm{C}$ for $7 \mathrm{~min}$. The amplicon was electrophoretically analyzed through $1.5 \%$ agarose gels, and visualized with a UV transilluminator after ethidium bromide staining (Sambrook and Russell, 2001). The intensities of the targets and $E F-1 \alpha$ were quantified from the gel photograph using the Quantity One software (BioRad), and relative expression levels of investigated transcripts (intensity of targets/intensity of $E F-1 \alpha$ ) in all experimental groups of $P$. monodon were statistically tested using analysis of variance (ANOVA), followed by the Duncan's new multiple range test. Results were considered significant when $\mathrm{p}<0.05$. The ovaries from five groups of shrimp (juveniles and stages I, II, III and IV broodstock, $N=4$ for each group) were assayed for expression analysis.

\section{Results and Discussion}

An understanding of the roles of genes functionally involved in ovarian and oocyte development should ultimately lead to a plausible approach for inducing reproductive maturation in P. monodon. In this study, 220 and 232 clones, respectively, from the forward (cDNAs from stage
III ovaries as the tester and those from stage I ovaries as the driver; GenBank accession no. GW775090-GW775309) and reverse (cDNAs from stage I ovaries as the tester and those from stage III ovaries as the driver; GenBank accession no. GW775310-GW775541) SSH ovarian libraries of $P$. monodon were unidirectionally sequenced and 136 $(61.8 \%)$ and 133 (57.3\%) ESTs, respectively, significantly matched known sequences in GenBank $\left(E\right.$-value $<10^{-4}$, Tables 2 and 3). Homologues of thrombospondin (TSP; 39 ESTs accounting for $17.7 \%$ and 26 ESTs accounting for $11.2 \%$ of sequenced clones) and peritrophin (39 ESTs, $17.7 \%$ and 27 clones, $11.6 \%$ ) were abundantly represented in both libraries similar to results from analyses of the conventional cDNA library of vitellogenic ovaries of $P$. monodon (79 and 87 clones accounting for 7.5 and $8.3 \%$ of clones sequenced, respectively; Preechaphol et al., 2007).

Relatively high percentages of unknown transcripts were found in both the forward and reverse SSH ovarian libraries of $P$. monodon (84 and 99 ESTs accounting for $38.2 \%$ and $42.7 \%$, respectively; Tables 2 and 3 ). The percentage of unknown transcripts in these $\mathrm{SSH}$ libraries was greater than that in the conventional ovarian (308/1051 clones, 29.3\%; Preechaphol et al., 2007) and testicular (290/889 clones, 32.6\%; Leelatanawit et al., 2009) cDNA libraries but lower than those found in the forward (112/178 ESTs, 62.9\%) and reverse (87/187 ESTs, 46.5\%) SSH testicular libraries of $P$. monodon, respectively (Leelatanawit et al., 2008).

After sequence assembly, 16 contigs (from 97 ESTs) and 123 singletons were obtained for the forward and 14 contigs (from 142 ESTs) and 90 singletons for the reverse SSH libraries, respectively. In all, 229 transcripts (28 contigs from 251 transcripts and 201 singletons, i.e., 44.5\%) were obtained when both libraries were analyzed simultaneously, of which 109 significantly matched known genes in GenBank $\left(E\right.$-value $\left.<10^{-4}\right)$. Disregarding contigs repre- 
Table 2 - Examples of known transcripts excluding ribosomal proteins found in the forward ovarian SSH library (cDNAs from stage III ovaries as the tester and those from stage I ovaries as the driver) of $P$. monodon.

\begin{tabular}{|c|c|c|c|c|}
\hline Transcript* & Species & Accession number & $E$-value & Size (bp) \\
\hline Peritrophin 2 & Penaeus monodon & AAM44050.1 & $5 \times 10^{-86}$ & 454 \\
\hline Peritrophin 1 & Penaeus monodon & AAM44049.1 & $4 \times 10^{-41}$ & 381 \\
\hline Thrombospondin & Penaeus monodon & AAN17670 & $1 \times 10^{-107}$ & 563 \\
\hline Thrombospondin & Marsupenaeus japonicus & BAC92764.1 & $3 \times 10^{-44}$ & 502 \\
\hline Keratinocyte-associated protein 2 & Rattus norvegicus & NP_001099914.1 & $8 \times 10^{-25}$ & 470 \\
\hline Eukaryotic translation initiation factor 2 , subunit 2 beta & Rattus norvegicus & AAH62402.1 & $7 \times 10^{-11}$ & 605 \\
\hline Ser/Thr Checkpoint kinase 1 (Chk1), CG17161-PA & Drosophila melanogaster & AAF53552 & $2 \times 10^{-22}$ & 417 \\
\hline $\begin{array}{l}\text { Methionyl-tRNA formyltransferase, mitochondrial precur- } \\
\text { sor (MtFMT) }\end{array}$ & Homo sapiens & NP_640335 & $4 \times 10^{-7}$ & 483 \\
\hline Nucleolin & Xenopus laevis & NP_001081557.1 & $8 \times 10^{-4}$ & 380 \\
\hline Eukaryotic initiation factor eIF-4A & Marsupenaeus japonicus & BAB78485 & $1 \times 10^{-41}$ & 279 \\
\hline $26 S$ proteasome regulatory subunit rpn 2 & Culex quinquefasciatus & XP_001862500 & $3 \times 10^{-52}$ & 468 \\
\hline Cytochrome c oxidase polypeptide IV & Bombyx mori & NP_001073120.1 & $3 \times 10^{-38}$ & 405 \\
\hline Hypothetical protein DKFZp434J1672.1 & Homo sapiens & $\overline{\mathrm{CAB}} 63724$ & $6 \times 10^{-24}$ & 525 \\
\hline Coatomer protein complex, subunit beta & Gallus gallus & NP_001006467.1 & $1 \times 10^{-67}$ & 588 \\
\hline Chaperonin containing T-complex polypeptide 1 & Carassius auratus & BAA89277 & $8 \times 10^{-44}$ & 627 \\
\hline ATP synthase oligomycin sensitivity conferral protein & Toxoptera citricida & AAU84928 & $3 \times 10^{-9}$ & 538 \\
\hline Cyclin $A$ & Asterina pectinifera & BAA14010 & $4 \times 10^{-42}$ & 368 \\
\hline Non-muscle myosin-II heavy chain & Apis mellifera & XP_393334 & $8 \times 10^{-99}$ & 712 \\
\hline $\begin{array}{l}\text { Procollagen-proline, 2-oxoglutarate 4-dioxygenase (pro- } \\
\text { tein disulfide isomerase-associated 1) }\end{array}$ & Xenopus tropicalis & CAJ83276 & $2 \times 10^{-47}$ & 663 \\
\hline Chaperonin containing $T C P 1$, subunit 7 & Danio rerio & NP_775355.1 & $4 \times 10^{-24}$ & 249 \\
\hline Isocitrate dehydrogenase 2 & Tribolium castaneum & EFA04299 & $1 \times 10^{-37}$ & 231 \\
\hline CD53 antigen & Homo sapiens & NP_001035122.1 & $4 \times 10^{-04}$ & 394 \\
\hline Calreticulin & Galleria mellonella & BAB79277 & $5 \times 10^{-103}$ & 714 \\
\hline DNA replication licensing factor $m \mathrm{~cm} 2$ & Xenopus tropicalis & AAH75567 & $2 \times 10^{-47}$ & 490 \\
\hline RNA binding motif protein 4 & Aedes aegypti & XP_001657237.1 & $6 \times 10^{-38}$ & 563 \\
\hline Domino isoform D, CG9696-PD & Apis mellifera & XP_396786 & $9 \times 10^{-61}$ & 350 \\
\hline $\begin{array}{l}\text { Eukaryotic translation initiation factor } 2 B \text {, subunit } 5 \text { epsi- } \\
\text { lon, isoform } 3\end{array}$ & Macaca mulatta & XP_001103944 & $5 \times 10^{-32}$ & 713 \\
\hline Translation initiation factor & Anopheles gambiae & CAD27760.1 & $2 \times 10^{-66}$ & 708 \\
\hline Secreted nidogen domain protein & Strongylocentrotus purpuratus & XP_001196268.1 & $8 \times 10^{-09}$ & 466 \\
\hline $\begin{array}{l}\text { Carbamoyl-phosphate synthetase } 2 \text {, aspartate } \\
\text { transcarbamylase, and dihydroorotase }\end{array}$ & Danio rerio & NP_001009884.1 & $4 \times 10^{-18}$ & 611 \\
\hline DEAD (Asp-Glu-Ala-Asp) box polypeptide 5 & Tribolium castaneum & XP_972501.1 & $3 \times 10^{-07}$ & 354 \\
\hline Deleted in malignant brain tumors 1 & Strongylocentrotus purpuratus & XP_001180356.1 & $2 \times 10^{-04}$ & 486 \\
\hline $\begin{array}{l}\text { ATPase, } H+\text { transporting, lysosomal accessory protein } \\
\text { 2, CG8444-PA }\end{array}$ & Tribolium castaneum & XP_973593.1 & $1 \times 10^{-07}$ & 562 \\
\hline Kinesin-like protein 2 & Ciona intestinalis & NP_001011659 & $5 \times 10^{-04}$ & 449 \\
\hline Elongation factor-1 alpha & Libinia emarginata & AAC03149 & $3 \times 10^{-102}$ & 713 \\
\hline Chromosome-associated protein, CG9802-PA, isoform A & Apis mellifera & XP_393700 & $2 \times 10^{-74}$ & 652 \\
\hline CWF19-like 2, cell cycle control & Xenopus tropicalis & NP_001039121.1 & $1 \times 10^{-58}$ & 600 \\
\hline Myosin II essential light chain & Tribolium castaneum & XP_973734 & $6 \times 10^{-15}$ & 516 \\
\hline Gastrula zinc finger protein XLCGF57.1 & Danio rerio & XP_001344037.1 & $4 \times 10^{-30}$ & 568 \\
\hline SJCHGC09076 protein & Schistosoma japonicum & AAW26562 & $6 \times 10^{-06}$ & 559 \\
\hline Citrate synthase & Aedes aegypti & EAT45772.1 & $4 \times 10^{-75}$ & 478 \\
\hline Zinc finger protein 146 & Strongylocentrotus purpuratus & XP_788425.2 & $2 \times 10^{-20}$ & 654 \\
\hline Sec23 protein & Drosophila melanogaster & NP_730978.1 & $6 \times 10^{-63}$ & 465 \\
\hline Elongation factor-2 & Libinia emarginata & AAR01298 & $8 \times 10^{-82}$ & 538 \\
\hline Hypothetical protein TTHERM_00449680 & Tetrahymena thermophila & XP_001013363.1 & $2 \times 10^{-10}$ & 506 \\
\hline Calreticulin & Bombyx mori & AAP50845.1 & $1 \times 10^{-128}$ & 695 \\
\hline$R N A$-binding protein 5 & Apis mellifera & XP_394165.3 & $4 \times 10^{-43}$ & 713 \\
\hline Mitochondrial ATP synthase e chain & Aedes albopictus & AAV90734 & $9 \times 10^{-16}$ & 403 \\
\hline Zgc: 113377 & Danio rerio & NP_001025397 & $4 \times 10^{-29}$ & 697 \\
\hline Inhibitor of Bruton agammaglobulinemai tyrosine kinase & Canis familiaris & XP_539018.2 & $2 \times 10^{-12}$ & 634 \\
\hline
\end{tabular}

*Accession no. GW775090-GW775309 for ESTs from the forward SSH library. 
Table 3 - Examples of known transcripts excluding ribosomal proteins found in the reverse ovarian SSH library (cDNAs from stage I ovaries as the tester and those from stage III ovaries as the driver) of $P$. monodon.

\begin{tabular}{|c|c|c|c|c|}
\hline Transcript* & Species & Accession number & $E$-value & Size (bp) \\
\hline Peritrophin 1 & Penaeus monodon & AAM44049.1 & $2 \times 10^{-53}$ & 412 \\
\hline Peritrophin 2 & Penaeus monodon & AAM44050.1 & $1 \times 10^{-72}$ & 406 \\
\hline Thrombospondin & Penaeus monodon & AAN17670 & $3 \times 10^{-63}$ & 368 \\
\hline Thrombospondin & Marsupenaeus japonicus & BAC92764.1 & $9 \times 10^{-61}$ & 405 \\
\hline Translation initiation factor eIF $4 A$ & Spisula solidissima & AAK85401 & $1 \times 10^{-47}$ & 326 \\
\hline CG10527-like methyltransferase & Mesobuthus gibbosus & CAE53527.1 & $1 \times 10^{-28}$ & 458 \\
\hline Selenoprotein M precursor & Homo sapiens & NP_536355.1 & $7 \times 10^{-24}$ & 560 \\
\hline $\begin{array}{l}\text { Stress- } 70 \text { protein, mitochondrial precursor ( } 75 \mathrm{kDa} \text { glu- } \\
\text { cose-regulated protein) }\end{array}$ & Gallus gallus & NP_001006147.1 & $1 \times 10^{-26}$ & 577 \\
\hline Neuralized protein & Drosophila virilis & AAB60619.1 & $4 \times 10^{-27}$ & 575 \\
\hline Secreted nidogen domain protein & Strongylocentrotus purpuratus & XP_001196268.1 & $3 \times 10^{-6}$ & 480 \\
\hline Thioesterase superfamily member 2 & Gallus gallus & XP_419092.1 & $3 \times 10^{-13}$ & 511 \\
\hline Hypothetical protein MGC75603 & Xenopus tropicalis & NP_989388 & $2 \times 10^{-6}$ & 642 \\
\hline Carbonyl reductase & Plecoglossus altivelis & BAB92960 & $2 \times 10^{-20}$ & 589 \\
\hline Ovarian lipoprotein receptor & Penaeus semisulcatus & AAL79675 & $4 \times 10^{-17}$ & 618 \\
\hline Allatotropin neuropeptide precursor & Spodoptera frugiperda & CAD98809.1 & $6 \times 10^{-9}$ & 402 \\
\hline Chitin deacetylase-like 9, CG15918-PA & Drosophila melanogaster & NP_611192.1 & $1 \times 10^{-17}$ & 353 \\
\hline Replication factor C/activator 1 subunit & Gallus gallus & AAA20552.1 & $5 \times 10^{-58}$ & 583 \\
\hline Nuclease diphosphate kinase B & Danio rerio & AAF60971 & $9 \times 10^{-34}$ & 430 \\
\hline Acyl-CoA synthase & Oceanicola batsensis & ZP_01000658.1 & $9 \times 10^{-51}$ & 518 \\
\hline $70 \mathrm{kD}$ heat shock-like protein & Procambarus clarkia & $\overline{\mathrm{ABC}} 01063$ & $1 \times 10^{-103}$ & 692 \\
\hline Signal sequence receptor & Bombyx mori & NP_001091760.1 & $3 \times 10^{-04}$ & 600 \\
\hline ATP synthase, CG11154-PA isoform A & Apis mellifera & XP_624156 & $6 \times 10^{-115}$ & 690 \\
\hline $\begin{array}{l}\text { Ubiquitin-like } 1 \text { activating enzyme E1B (SUMO-1 activat- } \\
\text { ing enzyme subunit 2) }\end{array}$ & Strongylocentrotus purpuratus & XP_001195210.1 & $4 \times 10^{-24}$ & 473 \\
\hline Ribonuclease P 40kDa subunit isoform 3 & Macaca mulatta & XP_001095772 & $6 \times 10^{-19}$ & 688 \\
\hline Selenophosphate synthetase (selenium donor protein) & Drosophila melanogaster & NP_725374.1 & $5 \times 10^{-103}$ & 710 \\
\hline Peptidylprolyl isomerase D & Danio rerio & NP_001002065.1 & $1 \times 10^{-24}$ & 589 \\
\hline Egalitarian & Drosophila melanogaster & AAF47054.4 & $3 \times 10^{-37}$ & 704 \\
\hline CCR4-NOT transcription complex, subunit 10 & Tribolium castaneum & XP_974052 & $2 \times 10^{-29}$ & 585 \\
\hline Protein phosphatase 2 c gamma & Aedes aegypti & EAT47444.1 & $2 \times 10^{-56}$ & 711 \\
\hline RNA polymerase I associated factor 53 isoform 1 & Canis familiaris & XP_531998 & $5 \times 10^{-16}$ & 710 \\
\hline Splicing factor, arginine/serine-rich 7 & Apis mellifera & XP_001122800 & $2 \times 10^{-41}$ & 633 \\
\hline Interleukin enhancer binding factor 2 & Mus musculus & NP_080650.1 & $4 \times 10^{-31}$ & 332 \\
\hline Nuclear autoantigenic sperm protein & Danio rerio & NP_956076.1 & & 700 \\
\hline Cyteine-rich with EGF-like domain 2, CG11377-PA & Tribolium castaneum & XP_971778.1 & $6 \times 10^{-25}$ & 510 \\
\hline Eukaryotic initiation factor $4 A$ & Callinectes sapidus & ABG67961 & $1 \times 10^{-64}$ & 569 \\
\hline ATP lipid-binding protein like protein & Marsupenaeus japonicus & BAB85212 & $9 \times 10^{-30}$ & 588 \\
\hline TRI1, CG7338-PA & Apis mellifera & XP_624169 & $3 \times 10^{-41}$ & 708 \\
\hline Ferritin & Litopenaeus vannamei & AAX55641.1 & $3 \times 10^{-31}$ & 306 \\
\hline Deleted in malignant brain tumors 1 & Strongylocentrotus purpuratus & XP_001180356.1 & $2 \times 10^{-05}$ & 713 \\
\hline $\begin{array}{l}\text { Transmembrane } 4 \text { superfamily member } 8 \text { isoform } 1 / \\
\text { Tetraspanin } 3\end{array}$ & Homo sapiens & NP_005715 & $3 \times 10^{-10}$ & 596 \\
\hline $\begin{array}{l}\text { Neutral alpha-glucosidase AB precursor (Glucosidase II } \\
\text { subunit alpha) }\end{array}$ & Sus scrofa & NP_999069.1 & $2 \times 10^{-49}$ & 707 \\
\hline Calreticulin precursor (CRP55) (Calregulin) & Oryctolagus cuniculus & NP_001075704.1 & $4 \times 10^{-19}$ & 300 \\
\hline Ataxin1 ubiquitin-like interacting protein & Gallus gallus & NP_001026544 & $5 \times 10^{-41}$ & 612 \\
\hline Hypothetical protein & Mus musculus & XP_922736.3 & $2 \times 10^{-15}$ & 403 \\
\hline$H L A$-B-associated transcript 3 & Apis mellifera & XP_001121013.1 & $8 \times 10^{-25}$ & 261 \\
\hline Cyclin B3, CG5814-PA & Apis mellifera & XP_397108 & $6 \times 10^{-46}$ & 427 \\
\hline Hypothetical protein cgd5_1220 & Cryptosporidium parvum & EAK88123.1 & $2 \times 10^{-08}$ & 460 \\
\hline Ring finger protein 2, CG15814-PA, isoform $A$ & Tribolium castaneum & XP_975438.1 & $9 \times 10^{-40}$ & 431 \\
\hline 2-Cys thioredoxin peroxidase & Aedes aegypti & AAL37254 & $1 \times 10^{-56}$ & 564 \\
\hline
\end{tabular}

*Accession no. GW775310-GW775541 for ESTs from the reverse SSH library. 
senting thrombospondin/peritrophin (8 contigs) and unknown proteins (12 contigs), 8 contigs matched ribosomal protein S6, elongation factor 1- $\alpha$, elongation factor 2 , calreticulin, ficolin, selenophosphate synthetase, $70 \mathrm{kDa}$ heat shock-like protein and a hypothetical protein, AGAP006171-PA.

The percent distribution of nucleotide sequences, according to GO categories of SSH ovarian cDNA libraries of P. monodon, was analyzed (Figure 1). In the category 'biological process', ESTs involved in metabolic processes were predominant (e.g. anaphase promoting complex subunit 11, S-adenosylmethionine synthetase and T-complex protein 1 subunit epsilon, i.e., $35.0 \%$ of the examined ESTs), followed by those involved in cellular processes (e.g. acidic p0 ribosomal protein, DNA replication licensing factor mcm 2 and coatomer protein complex subunit beta, i.e., $25.2 \%$ of the examined ESTs). Reproductionrelated ESTs (e.g RNA binding motif protein 4, neuralized protein, dynein and egalitarian) were found in $2.4 \%$ of the examined sequences of combined SSH data. This discovery rate is higher than that of the conventional ovarian cDNA libraries of P. monodon (1.7\%; Preechaphol et al., 2007).

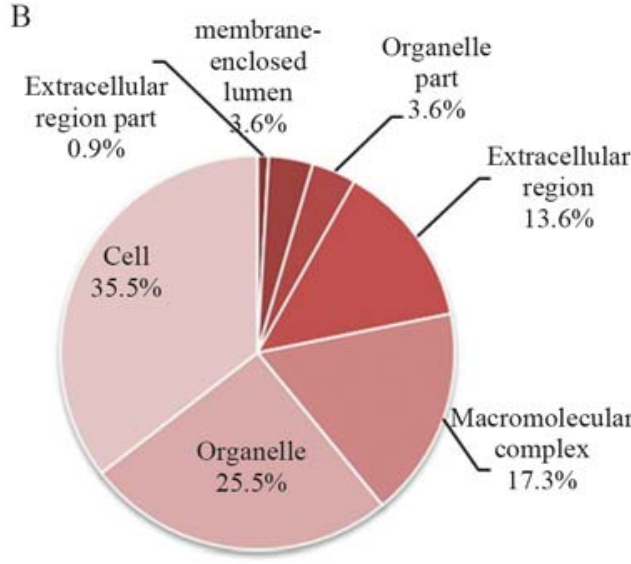

As for the category 'cellular component', ESTs functionally involved in the cell part (e.g. myosin II essential light chain, ATP synthase E chain and Ser/Thr checkpoint kinase 1, i.e., $35.5 \%$ of the examined ESTs) predominated, followed by those functionally displayed in organelles (e.g. selenoprotein $M$ precursor, keratinocyte-associated protein 2 and interleukin enhancer binding factor 2; 25.5\% of the examined ESTs).

In the category 'molecular function', ESTs involved in binding (e.g. carbonyl reductase, translation initiation factor eif-2b, RNA binding motif protein 5 isoform 9 and selenophosphate synthetase, i.e., $50.5 \%$ of the examined ESTs) predominated followed by those displaying catalytic activity (e.g. MGC80929 protein isoform 1, oncoprotein $n m 23$ and eukaryotic initiation factor $4 A$, i.e., $30.5 \%$ of the examined ESTs).

The highly organized eukaryotic cilia and flagella contain approximately 250 proteins (Inaba, 2003). They are constructed around evolutionarily conserved microtubulebased structures called axonemes (nine outer doublet microtubules, dynein arms, a central pair of microtubules and radial spokes) (Luck, 1984; Dutcher, 1995; King, 2000). Dynein is functionally related to the transport of var-
A
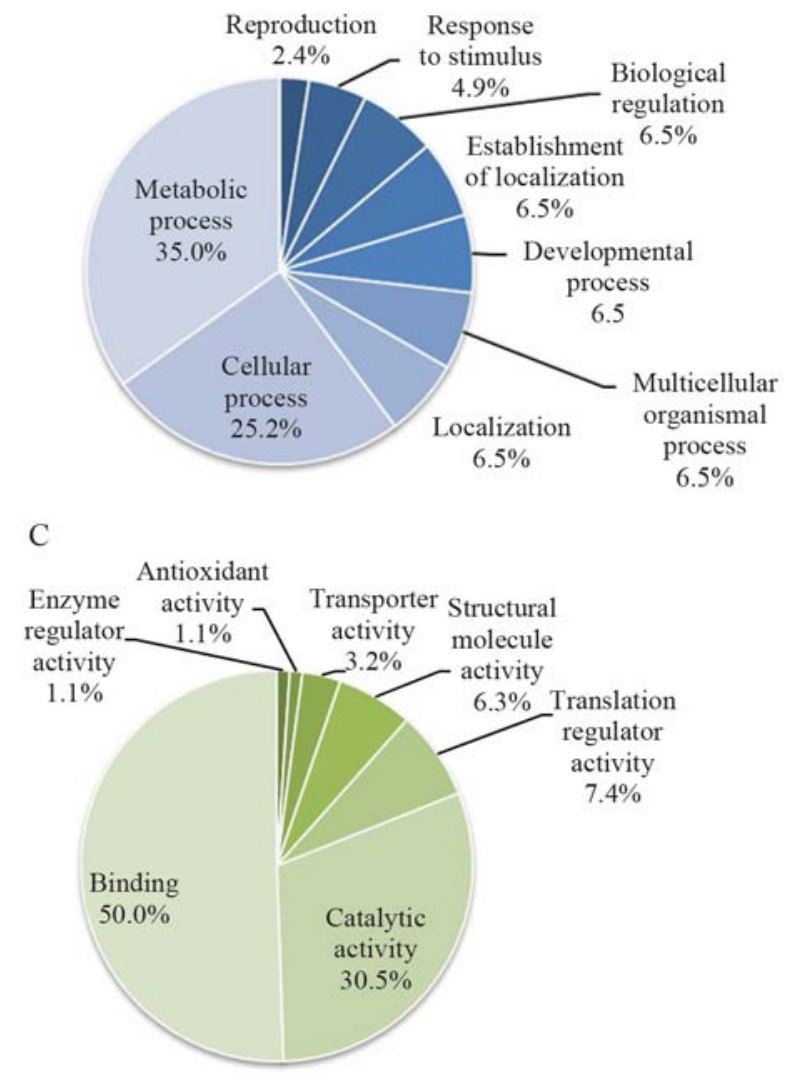

A

Figure 1 - The percent distribution of nucleotide sequences in the SSH ovarian cDNA library of $P$. monodon according to three principal GO categories: A, biological process; B, cellular components and C, molecular functions, respectively. 
ious cytoplasmic organelles (Aniento et al., 1993). In Drosophila, egalitarian binds to the dynein light chain. Point mutations that specifically inhibit Egl-Dlc association disrupt microtubule-dependant trafficking both to and within the oocyte, thereby resulting in a loss of oocyte fate maintenance and polarity (Carpenter, 1994).

The physiological role of carbonyl reductase was thought to be an NADPH-dependent reduction in a variety of endogenous and foreign carbonyl compounds. However, increasing evidence indicates its involvement in steroid metabolism. In ayu, its localization in ovaries, enzymatic characteristics and transcriptional increase with oocyte maturation, infer its additional function as 20ß-HSD in the production of maturation inducing hormones (MIH) (Tanaka et al., 2002).

The DNA replication (or origin) licensing system is prominant in ensuring precise duplication of the genome in each cell cycle, besides being a powerful regulator of metazoan cell proliferation (Eward et al., 2004). The protein kinase Chk1 plays a role in checkpoint control. Recombinant Xenopus Chk1 phosphorylates the mitotic inducer $\mathrm{Cdc} 25$ in vitro at multiple sites. Nevertheless, only XChk1-catalyzed phosphorylation of Cdc25 at Ser-287 is sufficient to confer the binding of 14-3-3 proteins (Kumagai et al., 1998). Moreover, the meiotic maturation of oocytes is regulated by the maturation promoting factor (MPF), a complex of cdc2 (Cdk1), cyclin B and other Cdk/cyclin complexes (Kobayashi et al., 1991; Kishimoto, 1999, 2003). Chk1-dephophorylated Cdc25 activates MPF, thereby causing meiotic resumption in oocytes (Kishimoto, 2003).

Recently, the full length cDNA of keratinocyteassociated protein 2 was isolated in the Pacific white shrimp (Litopenaeus vannamei), although the function of this protein is still unknown. Moreover, its expression was altered following infection by the White Spot Syndrome Virus, WSSV (Clavero-Salas et al., 2007).

The full length cDNAs of anaphase promoting complex subunit 11 (biological process GO:0008152; GenBank accession no. GW775392) and selenoprotein M precursor (cellular component GO:0005783; GenBank accession no. GW775333) were hereby reported and identified for the first time in penaeid shrimp.

The anaphase promoting complex subunit 11 of $P$. monodon (PmAPC11) was $600 \mathrm{bp}$ in length, and consisted of an ORF of $255 \mathrm{bp}$ corresponding to a polypeptide of 84 amino acids, with 5' and 3' UTRs of 1 and $387 \mathrm{bp}$, respectively (Figure 2A). The closest similar sequence to PmAPC11 was the anaphase promoting complex subunit 11 homolog of Tribolium castaneum $\left(E\right.$-value $\left.=1 \times 10^{-41}\right)$. The predicted molecular mass and $\mathrm{p} I$ of the deduced PmAPC11 was $9.84 \mathrm{kDa}$ and 7.99 , respectively. Activation of the anaphase-promoting complex (APC) by Cdc20 enables anaphase initiation and exit from mitosis (Kramer $e t$ al., 1998; Lorca et al., 1998).
A

CATGAAGGTGAAGATTAAATCCTGGACGGGATTGGCTACATGTCGGTGGTTGGCTAATGA 60 $\begin{array}{llllllllllllllllllllllll}M & K & V & K & I & K & S & W & T & G & \text { L } & \text { A } & \text { T } & \text { C } & \text { R } & \text { W } & \text { L } & \text { A } & \text { N } & \text { D } & 20\end{array}$ TGACAGTTGTGGCATTTGTAGGATGCCCTTTGATGGATGCTGCTCAGATTGTAGGTTGCC 120 $\begin{array}{lllllllllllllllllllll}D & S & C & G & I & C & R & M & P & F & D & G & C & C & S & D & C & R & L & P & 40\end{array}$ AGGTGATGACTGCCCACTAGTGTGGGGCCAGTGCTCTCACTGTTTCCATATTCACTGCAT 180 $\begin{array}{llllllllllllllllllllll}G & \text { D } & \text { D } & C & \text { P } & \text { L } & \text { V } & \text { W } & G & \mathcal{Q} & \text { C } & \text { S } & \text { H } & \text { C } & \mathbf{F} & \text { H } & \text { I } & \text { H } & \text { C } & \text { I } & 60\end{array}$ TATGAAGTGGCTTCAATCT CAACAGCTTCACCAGCAGTGTCCAATGTGTCGGCAAGAGT 240

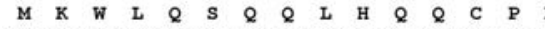
GAAGTTTAAAGAATAACCTTTAAAATACTTTATATTTTGTTTACTGTTTCAAGACTCATA 300 GTTGTGGGAGGAGTAAATTTGATGATTTGAAAGATATGGGTCACTCAAAGCATAGGGAC 360 TCGTCATCTGAGTCATTGGAGGAAAAACATAGAAGGAAACACAAAAAAAAAGCATAGGGAC 420

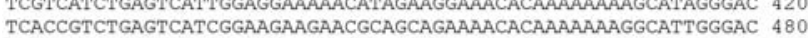
TCGTCGTCTGAATCTTTGGAGGAAGGACGTAGAAGAAGACACAAAAAAAGGCATAGGGAC 540 TCATCATCTGAGTCTTCGGAGGAAGAGCGCAGAAGAAAACACGAAAAAAAAAAAAAAAAAAA 600

B

GCAACCATGGCGAAAAGGAGTCTCCGGCTCCTCCTCCTGCTGGGGGTAGCGTTCTCTTAT 60 $\begin{array}{lllllllllllllllllll}M & \text { A } & \text { K } & \text { R } & \text { S } & \text { L } & \text { R } & \text { L } & \text { L } & \text { L } & \text { L } & \text { L } & \text { G } & \text { V } & \text { A } & \text { F } & \text { S } & \text { Y } & 18\end{array}$ ACCCTCGCTGAAGAATTACGT GAGACTGATATTGCCAAGGCTCGCGTTGAGAGCTGCGGT 120 GGATGACGTTTGAACAGCCTCCCCGAGGTGAHGAMATTCATCCACGAGGACATCCCACTC 180 I H E D I P I 58 TTCCATAATGCCAAGTTCAAGCAGATAGGAGGTGCTCCTCCAGAACTGGTTCTTCTCAAT 240 F $\mathrm{N}$

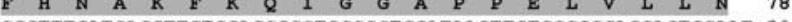

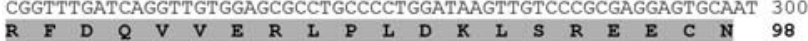
$\begin{array}{lllllllllllllllllllll}\mathbf{R} & \mathbf{F} & \mathbf{D} & \mathbf{Q} & \mathbf{V} & \mathbf{V} & \mathbf{E} & \mathbf{R} & \mathbf{L} & \mathbf{P} & \mathbf{L} & \mathbf{D} & \mathbf{K} & \mathbf{L} & \mathbf{S} & \mathbf{R} & \mathbf{E} & \mathbf{E} & \mathbf{C} & \mathbf{N} & \mathbf{9 8} \\ \text { AAAACTCATGCT GAAGAAGGGATTCTATAAAGAAGAAATTCACCCGACGAGGAAGTCCCTGAA } & 360\end{array}$ $\begin{array}{lllllllllllllllllllll}K & \text { L } & M & \text { L } & K & K & G & F & Y & K & K & N & \text { S } & \text { P } & \text { D } & \text { E } & \text { E } & \text { V } & \text { P } & \text { E } & 118\end{array}$ GAATACCTTAACGGGCCTTATAGAGAGAGGGAAGAACTGTAGATGAAACCTCGGGCAAAG 420

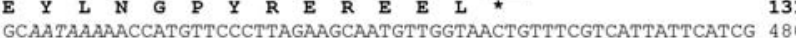
TGGAAACACATCTGTAGAAAATTCGAAGTCGTTAGACAGTGTCAGTATAATAGTCAACTA 540 GTTAAATCAGAGCAGATAGTACTTAGATTTTATTTGTAGAAATATCGAGTTTTTATATAA 600

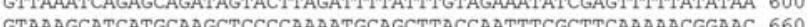
C ATGGAAGTACCGCACAAAATTCCGTCAGTAACATTTGTTACCAATATGGAGTCTCGGGGC 720 ACTCGAGGGAAGACACGATTTTCGACATGAAAAGGATCACGAAAAGGGTTCGACTCAAGT 780 GGTCCTTGGAAGCAAAGAATTATCTGGTTGTCCTTTCGAGTGTTTTGAGACGTTTGCATT 840 CCGTTGATTTTGTAGTTGTTTGAATAAAATTTAATTTTAAAAAAAAAAAAAAAAAAAAAAAAAA
900
A.AAA

Figure 2 - The full length cDNA and deduced protein sequences of PmAPC11 (600 bp, ORF of $255 \mathrm{bp}$ corresponding to a deduced polypeptide of 84 amino acids; GenBank accession no. GW775392) and PmSePM (904 bp, ORF of 396 bp corresponding to a deduced polypeptide of 131 amino acids; GenBank accession no. GW775333). The putative start and stop codons are illustrated in boldface and underlined. The predicted signal peptide and poly A additional signals of PmSePM are underlined and italicized and underlined, respectively. The predicted Sep15_SelM domain (positions 31-107) found in the deduced PmSePM protein is highlighted.

The selenoprotein $M$ precursor of $P$. monodon $(P m S e P M)$ was $904 \mathrm{bp}$ in length, and consisted of an ORF of $396 \mathrm{bp}$, corresponding to a polypeptide of 131 amino acids, and 5' and 3' UTRs of 6 and 502 bp, respectively (Figure 2B). It significantly matched the selenoprotein $M$ precursor of $L$. vannamei $\left(E\right.$-value $\left.=2 \times 10^{-58}\right)$. The predicted molecular mass and $\mathrm{p} I$ of the deduced PmSePM protein was $15.10 \mathrm{kDa}$ and 7.75 , respectively. PmSePM contained a signal peptide located between $\mathrm{A}_{21}$ and $\mathrm{E}_{22}$, as well as a Sep15_SelM domain (positions 31-107, $E$-value = $1.9 \times 10^{-34}$ ) that exerts the thiol-disulphide isomerase activity functionally involved in disulphide bond formation of proteins in the endoplasmic reticulum (ER) (Ferguson et al., 2006).

In addition, the EST representing selenophosphate synthetase, an enzyme involved in selenocysteine biosynthesis, was also identified. In humans, selenium deficiency leads to male infertility and susceptibility to viral infections. More than 20 selenoproteins have been identified in higher eukaryotes (Guimaraes et al., 1996; Rayman, 2000; Korotkov et al., 2002) but their functions in ovarian/oocyte 
development of $P$. monodon remain unknown. The analysis of baseline information, acquired as part of this study addresses the paucity of data and should provide a better understanding of reproductive maturation in cultured female P. monodon.

To address the functional involvement of various genes during ovarian development of P. monodon, the expression profiles of keratinocyte-associated protein 2, Ser/Thr Chk1, DNA replication licensing factor mcm2, $P m S e P M$ and egalitarian were examined by semiquantitative RT-PCR analysis. The control gene $(E F-1 \alpha)$ seemed to be comparably expressed in all the groups of samples examined, thereby inferring its acceptability for use in normalizing target gene expression. All transcripts were more abun-
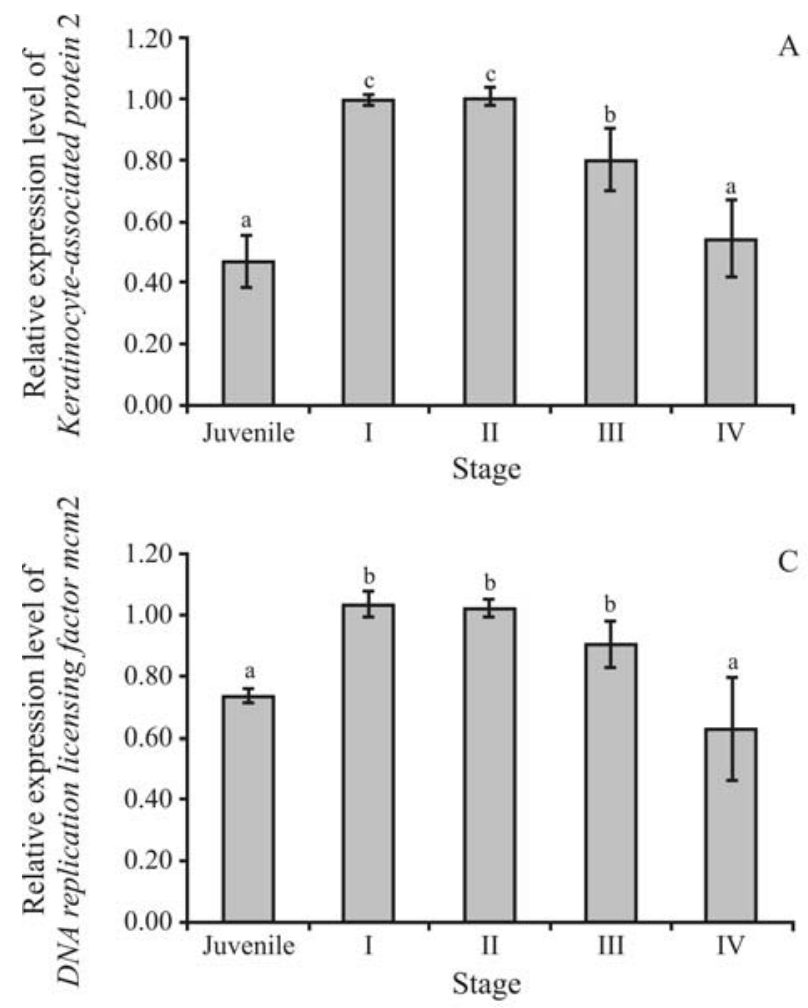

A
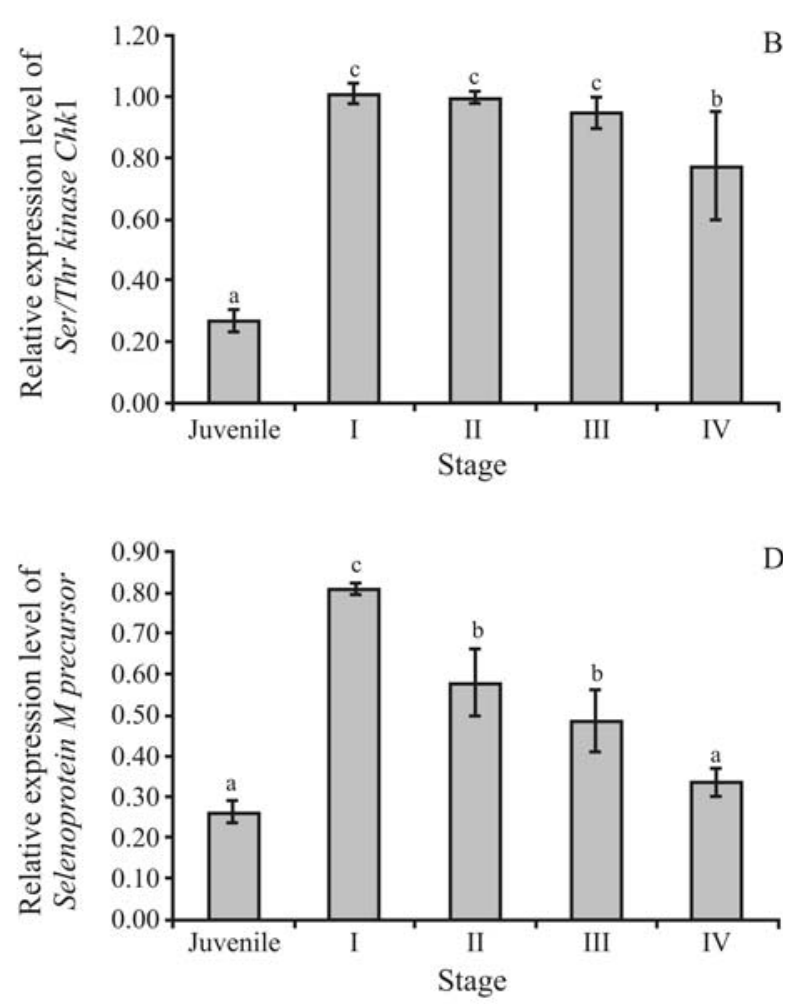

dantly expressed in the ovaries of broodstock than juveniles $(\mathrm{p}<0.05$, Figure 3). The expression level of PmSePM peaked in stage I (previtellogenic) of development (GSI $<1.5$ ), to progressively and significantly decrease in stages II (vitellogenic), III (cortical rod) and IV (mature) $(\mathrm{p}<0.05)$. Likewise, keratinocyte-associated protein 2 was initially down-regulated in stage III, and subsequently, stage IV $(\mathrm{p}<0.05)$. The expression of Ser/Thr Chk1, DNA replication licensing factor mcm 2 and egalitarian during stages I, II and III, was comparable $(\mathrm{p}<0.05)$, although down-regulated in the final stage of ovarian development in wild $P$. monodon broodstock $(\mathrm{p}<0.05$, Figure 3$)$.

In various animals, a wide variety of maternal mRNA is generally transcribed at the early oogenesis stage, to then

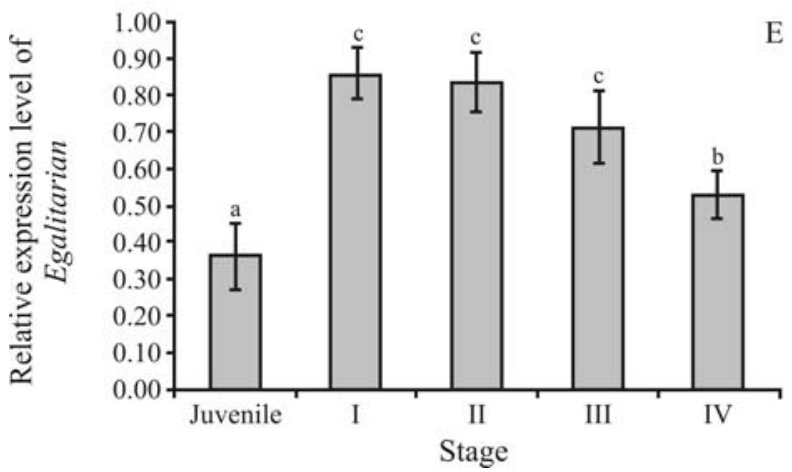

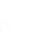


be stored in oocytes and carried into fertilized eggs (Qiu et al., 2008; Nishimura et al., 2009). Several reproductionrelated genes that are up-regulated during the ovarian development of $P$. monodon, for example, Ovarian-Specific Transcript 1 (Pm-OST1) and cyclin B (PmCyB), have been previously reported (Klinbunga et al., 2009; Visudtiphole et al., 2009). The down-regulation of keratinocyteassociated protein 2, Ser/Thr Chk1, DNA replication licensing factor mcm2, PmSePM and egalitarian implied that lower levels of these gene products may be necessary for the development and final maturation of $P$. monodon oocytes. The findings facilitate the possible use of RNA interference (RNAi) for studying their functional involvement in P. monodon ovarian development. Moreover, the expression profiles of keratinocyte-associated protein 2 and selenoprotein M precursor are potentially applicable as biomarkers to indicate degrees of reproductive maturation in the domesticated shrimp.

In this study, genes expressed in ovaries of $P$. monodon were identified by SSH analysis. The expression profiles of reproduction-related transcripts were examined. Further studies of the molecular mechanisms of those genes and proteins involved in controlling each stage of oocyte maturation should be carried out, to reach a better understanding of the reproductive maturation of $P$. monodon in captivity.

\section{Acknowledgments}

This research is financially supported by the National Center for Genetic Engineering and Biotechnology (BIOTEC), Thailand. Student grants (RP) were co-supported by The Royal Golden Jubilee PhD program, Thailand Research Funds (TRF) and the Commission of Higher Education Staff Development Project, Ministry of Education, Thailand.

\section{References}

Altschul SF, Gish W, Miller W, Myers EW and Lipman DJ (1990) Basic local alignment search tool. J Mol Biol 215:403-410.

Aniento F, Emans N, Griffiths G and Gruenberg J (1993) Cytoplasmic dynein-dependent vesicular transport from early to late endosomes. J Cell Biol 123:1373-1387.

Bailey-Brock JH and Moss SM (1992) Penaeid taxonomy, biology and zoogeography. In: Fast AW and Lester LJ (eds) Marine Shrimp Culture: Principles and Practices. Elsevier Science Publishers, Amsterdam, pp 9-23.

Benzie JAH (1998) Penaeid genetics and biotechnology. Aquaculture 164:23-47.

Carpenter AT (1994) Egalitarian and the choice of cell fates in Drosophila melanogaster oogenesis. Ciba Found Symp 182:223-254.

Clavero-Salas A, Sotelo-Mundo RR, Gollas-Galván T, Hernández-López J, Peregrino-Uriarte AB, Muhlia-Almazán A and Yepiz-Plascencia G (2007) Transcriptome analysis of gills from the white shrimp Litopenaeus vannamei infected with
White Spot Syndrome Virus. Fish Shellfish Immunol 23:459-472.

Conesa A, Götz S, Garia-Gómez JM, Terol J, Talón M and Robles M (2005) Blast2GO: A universal tool for annotation, visualization and analysis in functional genomics research. Bioinformatics 21:3674-3676.

Diatchenko L, Lau YC, Campbell AP, Chenchik A, Moqadam F, Huang B, Lukyanov S, Lukyanov K, Gurskaya N, Sverdlov ED et al. (1996) Suppression subtractive hybridization: A method for generating differentially regulated or tissuespecific cDNA probes and libraries. Proc Natl Acad Sci USA 93:6025-6030.

Dutcher SK (1995) Flagellar assembly in two hundred and fifty easy-to-follow steps. Trends Genet 11:398-404.

Eward KL, Obermann EC, Shreeram S, Loddo M, Fanshawe T, Williams C, Jung H-I, Prevost AT, Blow JJ, Stoeber K et al. (2004). DNA replication licensing in somatic and germ cells. J Cell Sci 117:5875-5886.

Ferguson AD, Labunskyy VM, Fomenko DE, Arac D, Chelliah Y, Amezcua CA, Rizo J, Gladyshev VN and Deisenhofer J (2006) NMR structures of the selenoproteins Sep15 and SelM reveal redox activity of a new thioredoxin-like family. J Biol Chem 281:3536-3543.

Guimaraes MJ, Peterson D, Vicari A, Cocks BG, Copeland NG, Gilbert DJ, Jenkins NA, Ferrick DA, Kastelein RA, Bazan JF et al. (1996) Identification of a novel selD homolog from eukaryotes, bacteria, and archaea: Is there an autoregulatory mechanism in selenocysteine metabolism? Proc Natl Acad Sci USA 93:15086-15091.

Huang X and Madan A (1999) CAP3: A DNA sequence assembly program. Genome Res 9:868-877.

Inaba K (2003) Molecular architecture of the sperm flagella: Molecules for motility and signaling. Zool Sci 20:1043-1056.

Kenway M, Macbeth M, Salmon M, McPhee C, Benzie J, Wilson K and Knibb W (2006) Heritability and genetic correlations of growth and survival in black tiger prawn Penaeus monodon reared in tanks. Aquaculture 259:138-145.

King SM (2000) The dynein microtubule motor. Biochim Biophys Acta 1496:60-75.

Kishimoto T (1999) Activation of MPF at meiosis reinitiation in starfish oocytes. Dev Biol 214:1-8.

Kishimoto T (2003) Cell-cycle control during meiotic maturation. Curr Opin Cell Biol 15:654-663.

Klinbunga S, Sittikankaew K, Yuvanatemiya V, Preechaphol R, Presertlux S, Yamano K and Menasveta P (2009) Molecular cloning and expression analysis of ovary-specific transcript 1 (Pm-OST1) of the giant tiger shrimp, Penaeus monodon. Zool Sci 26:783-790.

Kobayashi H, Minshull J, Ford C, Golsteyn R, Poon R and Hunt T (1991) On the synthesis and destruction of A- and B-type cyclins during oogenesis and meiotic maturation in Xenopus laevis. J Cell Biol 114:755-765.

Korotkov KV, Novoselov SV, Hatfield DL and Gladyshev VN (2002) Mammalian selenoprotein in which selenocysteine (Sec) incorporation is supported by a new form of Sec insertion sequence element. Mol Cell Biol 22:1402-1411.

Kramer ER, Gieffers C, Holzl G, Hengstschlager M and Peters JM (1998) Activation of the human anaphase-promoting complex by proteins of the CDC20/Fizzy family. Curr Biol 8:1207-1210. 
Kumagai A, Guo Z, Emami KH, Wang SK and Dunphy WG (1998) The Xenopus Chk1 protein kinase mediates a caffeine-sensitive pathway of checkpoint control in cell-free extracts. J Cell Biol 142:1559-1562.

Leelatanawit R, Klinbunga S, Hirono I, Aoki T and Menasveta P (2008) Suppression subtractive hybridization (SSH) for isolation and characterization of genes related to testicular development of the giant tiger shrimp Penaeus monodon. BMB Rep 41:396-402.

Leelatanawit R, Sittikankeaw K, Yocawibun P, Klinbunga S, Hirono I, Aoki T and Menasveta P (2009) Identification, characterization and expression of sex-related genes in testes of the giant tiger shrimp Penaeus monodon. Comp Biochem Physiol A 152:66-76.

Limsuwan C (2004) Diseases of Pacific white shrimp (Litopenaeus vannamei) cultured in Thailand. Proceeding of the JSPS-NRCT International Symposium Joint Seminar 2004: Management of Food Safety in Aquaculture and HACCP. Kasetsart University, Thailand, pp 36-41.

Lorca T, Castro A, Martinez A-M, Vigneron S, Morin N, Sigrist S, Lehner C, Dorée M and Labbé J-C (1998) Fizzy is required for activation of the APC/cyclosome in Xenopus egg extracts. EMBO J 17:3565-3575.

Luck DJL (1984) Genetic and biochemical dissection of the eukaryotic flagellum. J. Cell Biol 98:789-794.

Nishimura Y, Endo T, Kano K and Naito K (2009) Porcine Aurora A accelerates Cyclin B and Mos synthesis and promotes meiotic resumption of porcine oocytes. Anim Reprod Sci 113:114-124.

Pertea G, Huang X, Liang F, Antonescu V, Sultana R, Karamycheva S, Lee Y, White J, Cheung F, Parvizi B et al. (2003) TIGR gene indices clustering tools (TGICL): A software system for fast clustering of large EST datasets. Bioinformatics 19:651-652.

Preechaphol R, Leelatanawit R, Sittikhankaew K, Klinbunga S, Khamnamtong B, Puanglarp N and Menasveta P (2007) Expressed sequence tag analysis for identification and characterization of sex-related genes in the giant tiger shrimp Penaeus monodon. J Biochem Mol Biol 40:501-510.

Qiu G-F, Ramachandra RK, Rexroad CE and Yao J (2008) Molecular characterization and expression profiles of cyclin B1, $\mathrm{B} 2$ and $\mathrm{Cdc} 2$ kinase during oogenesis and spermatogenesis in rainbow trout (Oncorhynchus mykiss). Anim Reprod Sci 105:209-225.
Quackenbush LS (1992) Yolk synthesis in the marine shrimp, Penaeus vannamei. Comp Biochem Physiol A 103:711-714.

Rayman MP (2000) The importance of selenium to human health. Lancet 356:233-241.

Rosenberry B (2001) World Shrimp Farming 2001. Shrimp News International, San Diego, 316 pp.

Sambrook J and Russell DW (2001) Molecular Cloning: A Laboratory Manual. 3rd edition. Cold Spring Harbor Laboratory Press, New York.

Tanaka M, Nakajin S, Kobayashi D, Fukuda S, Guan G, Todo T, Senthilkumaran B and Nagahama Y (2002) Teleost ovarian carbonyl reductase-like $20 \beta$-hydroxysteroid dehydrogenase: Potential role in the production of maturation-inducing hormone during final oocyte maturation. Biol Reprod 66:1498-1504.

Visudtiphole V, Klinbunga S and Kirtikara K (2009) Molecular characterization and expression profiles of cyclin $A$ and cyclin $B$ during ovarian development of the giant tiger shrimp Penaeus monodon. Comp Biochem Physiol A 152:535-543.

Withyachumnarnkul B, Boonsaeng V, Flegel TW, Panyim S and Wongteerasupaya C (1998) Domestication and selective breeding of Penaeus monodon in Thailand. In: Felgel T (ed) Proceedings to the Special Session on Advances in Shrimp Biotechnology, The Fifth Asian Fisheries Forum: International Conference on Fisheries and Food Security Beyond the Year 2000. Chiengmai, Thailand, pp 73-77.

Wongprasert K, Asuvapongpatana S, Poltana P, Tiensuwan M and Withyachumnarnkul B (2006) Serotonin stimulates ovarian maturation and spawning in the black tiger shrimp Penaeus monodon. Aquaculture 261:1447-1454.

\section{Internet Resources}

SeqClean software: http://www.tigr.org/tdb/tgi/software/ (November 20, 2009).

RepeatMasker software, University of Washington Genome Center, Seattle: http://ftp.genome.washington.edu/cgi-bin/ RepeatMasker (November 20, 2009).

\section{Associate Editor: Klaus Hartfelder}

License information: This is an open-access article distributed under the terms of the Creative Commons Attribution License, which permits unrestricted use, distribution, and reproduction in any medium, provided the original work is properly cited. 